\title{
Molecular Typing and Epidemiology Profiles of Human Metapneumovirus Infection Among Children With Severe Acute Respiratory Infection in Huzhou, China
}

\section{Lei Ji}

Huzhou Center for Disease Control and Prevention

\section{Liping Chen}

Huzhou Center for Disease Control and Prevention

Deshun Xu ( $\nabla$ xds666092@126.com)

Huzhou Center for Disease Control and Prevention https://orcid.org/0000-0001-5580-1997

Xiaofang Wu

Huzhou Center for Disease Control and Prevention

\section{Research Article}

Keywords: Human metapneumovirus, Respiratory tract infection, Epidemiology

Posted Date: July 8th, 2021

DOI: https://doi.org/10.21203/rs.3.rs-538269/v2

License: (c) (i) This work is licensed under a Creative Commons Attribution 4.0 International License.

Read Full License 


\section{Abstract}

Background: Human metapneumovirus (hMPV) is one of the important pathogens of infant respiratory tract infection. However, the molecular epidemiological information relating to hMPV among children hospitalized patients with severe acute respiratory infection (SARI) have not been thoroughly studied as yet. To investigate the infection status and genotypes of hMPV among children hospitalized patients with SARI from January 2016 to December 2020 in Huzhou, China.

Methods: A nasopharyngeal flocked swab, nasal wash or combination of nasopharyngeal swab and oropharyngeal swab samples were collected from children with SARI in Huzhou from January 2016 to December 2020. RT-PCR was used to detect the nucleic acid of hMPV. F gene was amplified and sequenced for the positive nucleic acid samples of hMPV. The obtained gene sequences were analyzed by MEGA software (version 7.0). Epidemiological data were analyzed using Microsoft Excel 2010 and service solutions (SPSS) 21.0 software.

Results: A total of 1133 children with SARI were collected from 2016 to 2020 . Among them, 56 cases were positive for hMPV nucleic acid, with a positive rate of $4.94 \%$. Children under 5 years old accounted for $85.71 \%$ of the total positive cases. Higher activity of hMPV infection could be seen in the period in Spring and Winter. and the main epidemic months were December and January-March. The F gene sequences of 28 strains of hMPV were obtained by co-sequencing. Phylogenetic analysis showed that there were A1, B1 and B2 genotypes of hMPV prevalent in Huzhou, and the dominant genotype of hMPV during our study period varied according to surveillance year.

Conclusions: HMPV is one of the important pathogens causing acute respiratory virus infection in children in Huzhou, with high incidence in winter and spring seasons and children under the age of 5『A1, $\mathrm{B} 1$ and $\mathrm{B} 2$ are three prevalent genotypes.

\section{Background}

Human metapneumovirus (hMPV), frst identifed in 2001, is a major viral respiratory pathogen that worldwide reported[1],Since then, scholars around the world have reported its infection [2,3] .hMPV is one of the important pathogens of infant respiratory tract infection $\square$ about half of all children are infected by

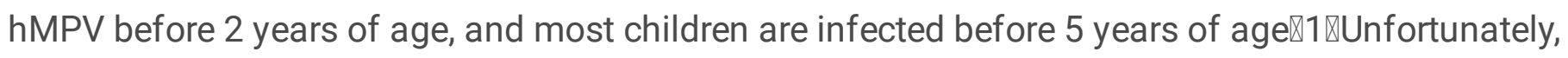
individuals infected with hMPV usually do not develop lifelong immunity against this virus, and reinfection occurs frequently[1,4]. It is a serious threat to the health of infants under five years old, the elderly and people with immunodeficiency. Global epidemiological studies have shown that most children have been infected with hMPV by the age of five[1]. hMPV infection cannot be distinguished from other respiratory viral infections by its symptoms alone, hMPV can cause upper or lower respiratory tract infections, mainly manifested by cough, expectoration, wheezing, shortness of breath, runny nose, bronchitis, asthmatic bronchitis, bronchiolitis, pneumonia, et al $₫ 5 \rrbracket$. hMPV is a non-segmented, negativestranded RNA virus belonging to the family Pneumoviridae. The viral RNA is approximately $13 \mathrm{~kb}$ in 
length, containing eight genes(N,P,M,F,M2,SH,G,and $\mathrm{L})$ coding for nine proteins $₫ 5 \rrbracket$. The evolutionary analysis of multiple genes showed that hMPV was divided into two different genotypes, $A$ and $B$, and within each genotype there were two different subtypes (A1, A2 and B1, B2) [6]. The fusion protein expressed by $\mathrm{F}$ gene is the main antigen of hMPV and is the most commonly used gene for typing at present [7]. Different subtypes of hMPV can be prevalent at the same time, and the dominant types of hMPV can appear alternately with time. At present, the main prevalent subtypes are A2, B1 and B2 genotypes $₫ 8-10 \rrbracket$. So far, the correlation between different subtypes of hMPV infection and disease severity has not been determined 111 . Recently, infections of hMPV were increasingly reported in Asian countries $₫ 8,12-19 \rrbracket$, with novel variants of $h M P V$ emerging $₫ 18,19 \rrbracket$, highlighting the risks of $h M P V$ epidemics in these regions. However, there is currently no effective vaccine or antiviral drug for hMPV 20区. Therefore, it is important to understand the prevalence and pathogenesis of hMPV for prevention and treatment. The in-depth analysis of the genomic structure of different genotypes of hMPV is helpful to understand its genetic background and evolutionary status, and provide a molecular basis for epidemiological investigation, prevention and treatment of hMPV.

The genetic evolution and transmission of hMPV are critical to epidemic control but have not been thoroughly studied as yet. Here, we conducted a fve-year study on hMPV in Huzhou, the most populous city in China, from January 2016 to December 2020.

\section{Materials And Methods}

Patients and Clinical sample Patients suspected of having acute respiratory tract infections were enrolled according to these criteria $₫ 21 \rrbracket$ : the onset of the disease has a history of fever $\left(>38^{\circ} \mathrm{C}\right)$, accompanied by sore throat, cough, expectoration, or dyspnoea/tachypnoea, and the onset does not exceed 10 days. All the samples of this study were obtained from local SARI surveillance sentinel hospital, the First People's Hospital of Huzhou was responsible for sample collection from surveillance cases. A total of 1133 patients were enrolled from January 2016 to December 2020. A nasopharyngeal flocked swab, nasal wash or combination of nasopharyngeal swab and oropharyngeal swab was collected from each children hospitalized patients, placed in $3 \mathrm{ml}$ viral transport medium, and stored at $-80^{\circ} \mathrm{C}$ prior to laboratory screening.

\section{RNA extraction and rRT-PCR}

Viral RNA is extracted from $200 \mu$ of the clinical specimens using a QIAamp viral RNA mini kit (Qiagen, Hilden, Germany) according to the manufacturer's instructions. The RNA extracts were subjected directly to reverse transcription (RT)-PCR (Polymerase Chain Reaction) or stored at $-70{ }^{\circ} \mathrm{C}$. Real-time RT-PCR (qPCR) was performed using nucleic acid detection kit for hMPV (Zhuocheng, Baijin, China) with the ABI Q7 (Applied Biosystems). The reaction was conducted according to the manufacturer's instructions with total volume of $20 \mu \mathrm{L}$.

\section{PCR amplifcation of $F$ protein genes}


For hMPV positive samples, the viral RNA was reverse transcribed into cDNA using the One Step RNA PCR Kit (TaKaRa Biotechnology Dalian, China, CAT: DRR057A). Then the F genes was amplified using traditional PCR with primers newly designed in this study. Primers used for $F$ gene sequencing were: outer primers set hMPV-F-F1-5'-CAATGCAGGTATAACACCAGCAATATC-3' and hMPV -F-R1 5'-

GCAACAATTGAACTGATCTTCAGGAAAC-3', and inner primers set hMPV-F-F2-5'-

ACATGCCAACATCTGCAGGACAAATAAAAC-3' and hMPV-F- R2 5'- ACATGCTGTTCACCTTCAACTTTGC-3'. And the amplification conditions as follows: $95^{\circ} \mathrm{C}$ for $5 \mathrm{~min}$, followed by 35 cycles of $95^{\circ} \mathrm{C}$ for $30 \mathrm{sec}$, $57^{\circ} \mathrm{C}$ for $30 \mathrm{sec}$, and $72^{\circ} \mathrm{C}$ for $1.5 \mathrm{~min}$, and a final step at $72^{\circ} \mathrm{C}$ for $5 \mathrm{~min}$. After amplification, $5 \mu \mathrm{L}$ of the PCR products was visualized by agarose gel electrophoresis. The residual PCR products were purified using a QIA quick PCR purification kit (Qiagen, Leusden, The Netherlands), and the purified products were sequenced directly at both ends with amplification primers by TaKaRa Biotechnology (Dalian, China).

\section{Phylogenetic analysis}

The hMPV reference strains sequences (LC337720, GU048745, MT118718, LC337745, GU048746, JQ745069, KF192752, GU048745, GU048741) of A1, B1 and B2 were downloaded from Genbank. The phylogenetic analysis was constructed using the neighbor-joining algorithm with the Kimura twoparameter model and supported statistically by bootstrapping with 1000 replicates with MEGA software (version 7.0) with 1000 bootstrap replicates.

Statistical analysis Microsoft Excel 2010 and SPSS22.0 software was used for statistical processing, and the count data rate (\%) was expressed. Chi-square test was used for comparison between groups. Pvalues $<0.05$ indicates a statistically significant difference.

\section{Nucleotide sequence accession numbers}

The GenBank accession numbers for sequences obtained in this study are MZ215789-MZ215816.

\section{Results}

\section{Epidemic characteristics of hMPV}

A total of 1133 nasopharyngeal swab samples of hospitalized children with SARI were collected from January 2016 to December 2020, and 56 samples were positive for hMPV, with the detection rate of 4.94\%(56/1133). among the 56 hMPV-infected patients, 33 (58.93\%) were male and $23(41.07 \%)$ were female. There was no significant difference in the positive rate between male and female $(P=0.395)$. The positive rate of hMPV infection in hospitalized children with SARI was $6.03 \%$ in the 0区year-old group, the positive rate of 1 \year-old group was $4.43 \%$, the positive rate of $3 \otimes$ years $4.61 \%$, the positive rate of $5 \rrbracket$ year-old group was $4.76 \%$, the positive rate of $7 \otimes 14$ year-old group was $4.39 \%$. There were no significant differences in hMPV detection rates among different age groups $(P=0.890)$. (Table1)

In the years 2016-2019, the hMPV detection rate was approximately even, ranging from $4.72 \%$ to $5.80 \%$, similar with the rate detected in central and south China. An apparent low frequency of circulation 
of hMPV in the year 2020 was observed, with a low positive rate of $1.79 \%$. The seasonal distribution showed that hMPV circulated predominantly in the period in Spring and Winter (Fig. 1). In contrast, lower activity of hMPV infection were observed during Summer and autumn, when the average detection rate was only $1.16 \%$ and $1.96 \%$ respectively.

\section{HMPV genotyping and phylogenetic analysis}

The F gene amplification and sequencing were performed on 56 hMPV positive samples, and the F gene sequences of 28 strains of viruses were successfully obtained. Phylogenetic of reference strains and obtained in this study (MZ215789-MZ215816) analysis was performed using MEGA software (version 7.0) with 1000 bootstrap replicates, among which 6 strains were A1 genotype, 12 were B1 genotype, and 10 were B2 genotype. The predominant genotypes of hMPV during our study period varied according to surveillance year. Overall, 1 type (hMPV-B1) were detected in 2016, 2 different types were detected in 2017, including hMPV-B1 ( $n=3$ ) and HAdV-A1 ( $n=2)$, 3 different types were detected in 2018, including hMPV-B1 ( $n=1)$, hMPV-B2 $(n=3)$ and hMPV-A1 $(n=4), 2$ different types were detected in 2019, including hMPV-B1 ( $n=2)$ and hMPV-B2 ( $n=5), 1$ type (hMPV-B2) were detected in 2020. (Figure 2).

\section{Discussion}

At present, hMPV has been recognized as one of the important pathogens of respiratory tract infection in the world. The diseases caused by hMPV are not significantly different from other viral infections, ranging from mild upper respiratory tract infection to severe bronchopneumonia. Most of the clinical manifestations were cough, runny nose, fever and wheezing. Hypoxemia occurred in about 1/3 of the patients. Chest X-ray showed local infiltrating shadow of pulmonary lobes or infiltration around hilar lung and peritracheal cuff sign. It is estimated that $4-16 \%$ of acute respiratory tract infections are caused by hMPV $[22,23]$. The virus has attracted wide attention at home and abroad since it was first identified in 2001. In 2003, Zhu Runan et al [9] first reported the infection of hMPV in China, and then similar reports were successively reported in other places in China [10-12]. However, there has been no research report on the infection of hMPV in Huzhou at present.

The onset of hMPV infection has a certain seasonality, with most reports suggesting that it occurs in winter and spring. In the northern hemisphere peak hMPV disease occurrence is typically in winter and spring months of January to May $[24,25,26]$, while in the southern hemisphere peak prevalence is in the spring period of August to September [27]. This study showed that there was a statistically significant difference in the detection rate of hMPV among the positive cases in different months. The main epidemic months were November and January-March, and the epidemic season was winter and spring, which was consistent with the results reported by Jin $\mathrm{Yu}$ [13]. In addition, the results of this paper show that the hMPV detection rate was approximately in the years 2016-2019, but an apparent low frequency of circulation of hMPV in the year 2020, with a low positive rate of $1.79 \%$, which may be related to factors such as the emergence of the local 2019-nCoV in February 2020 in Huzhou, the increased awareness of crowd protection, the reduction in crowd gathering, and the suspension of kindergarten and school. 
Worldwide, hMPV prevalence in hospital inpatient or community studies, in children or elderly adults, varies widely from as low as $1.7 \%$ to as high as $17 \%$, with generally higher prevalence in outpatients compared to inpatients and, also, more in children younger than 5 years compared to older age groups $[28,29,30]$. In this study, 1133 cases of children with severe acute respiratory tract collected in Huzhou from 2016 to 2020 were tested for hMPV nucleic acid, and 56 cases were detected positive, with a positive rate of $4.94 \%$, indicating that hMPV is indeed one of the important pathogens causing severe acute respiratory tract infection in children in Huzhou. The total number of cases under 5 years old was $84.55 \%(958 / 1133)$, The number of positive cases was $85.71 \%(48 / 56)$; No significant difference was found between the two sexes in the infection of the virus, which was consistent with the report of $\mathrm{Xu}$ Meijia [14].

Studies have shown that two types of hMPV genotypes A and B can be prevalent together in the same season, and genotype $A$ is the most prevalent. The prevalence pattern of hMPV genotypes in the same region may change continuously in different years. Liu Shiwen reported [31] that there were A2, B1 and B2 genotypes of hMPV prevalent in Jiangxi, among which A2 genotype was the dominant genotype. Our monitoring data showed there are A1, B1 and B2 genotypes of hMPV prevalent in Huzhou area. The B1 genotype strain was the most prevalent types and has been detected every year (except in 2020), followed by $B 2$ and $A 1$, so we speculated that the B1 genotype strain is the main epidemic strain in Huzhou area. However, the genotypes of the endemic strains are different from year to year, and one or several endemic types exist simultaneously every year, there are no type of hMPV presented absolutely predominant during hMPV epidemic seasons.

Our study is limited by a single-site setting, small sample size, and especially the partial genotyping of detected hMPV. Genotyping was only successful for $50 \%(28 / 56)$ of hMPV infection cases. In the future research, we will gradually improve the research content, expand the detection range and quantity of samples, accumulate and analyze data, further evaluate the harm of hMPV-related diseases, and provide more scientific basis for the prevention and control of infection of this virus.

\section{Conclusions}

HMPV is one of the important pathogens of SARI in hospitalized children in Huzhou area. It usually occurs in winter and spring and children under 5 year old, and three genotypes A1,B1 and B2 are prevalent at the same time, no type of hMPV presented absolutely predominant during hMPV epidemic seasons, which brings great difficulties to the diagnosis and treatment of SARI children. Our results provide great significance to improve the prevention and control of hMPV in Huzhou area.

\section{Abbreviations}

SARI: Severe acute respiratory infections

hMPV: Human metapneumovirus 
qPCR: Real-time RT-PCR

\section{Declarations}

\section{Ethics approval and consent to participate}

This study was approved by the ethics committee of Huzhou Center for Disease Control and Prevention. Informed consent for the nasopharyngeal swabs was obtained from the patients or their guardians. This study was part of a routine laboratory-based investigation. No human experimentation was conducted. The only human materials used were nasopharyngeal swabs that had been sent to our laboratory for routine virological diagnosis.

\section{Declarations}

The authors declare that they have no competing interests.

\section{Funding}

No.

\section{Consent for publication}

Not applicable.

\section{Availability of data and materials}

The readers interested in using the data may contact the corresponding author.

\section{Authors' contributions}

LJ and DSX participated in the design of the study and performed the statistical analysis. DSX and LPC participated in the hMPV detection. XFW and DSX participated in the genomic amplification for genotyping. LPC and LJ participated in the sequence analysis and phylogenetic analysis. LPC drafted the manuscript. All authors read and approved the final manuscript.

\section{Acknowledgements}

We thank the staff of the First People's Hospital in Huzhou for collecting the samples.

\section{References}

1. Hoogen, B. Gvanden et al. A newly discovered human pneumovirus isolated from young children with respiratory tract disease. Nature Medicine7, 719-724 (2001) 
2. ZHU Ru-nan, QIAN Yuan囚DENG Ji, et al. Human metapneumovirus may associate with acute respiratory infections in hospitalized pediatric patients in Bering, China狈. Chin J Pediatr冈June 2003, 41(6):441-444.

3. Boivin G, Abed Y, Pelletier G, Ruel L, Moisan D, Cote S, Peret T C, Erdman D D, Anderson L J. Virological features and clinical manifestations associated with human metapneumovirus: a new paramyxovirus responsible for acute respiratory-tract infections in all age groups $\mathbb{J} \mathbb{J}$. J Infect Dis, 2002,186(9):13301334.

4. Ebihara,T.; Endo,R.; Kikuta,H.; Ishiguro,N.; Ishiko,H.; Hara,M.; Takahashi,Y.; Kobayashi,K. Human metapneumovirus infection in Japanese children. J.Clin.Microbiol.2004,42,126-132.

5. Van den Hoogen B G, Bestebroer T M, Osterhaus AD, Fouchier R A. Analysis of the genomic sequence

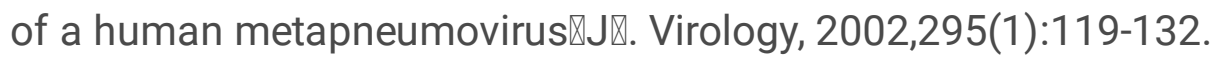

6. Van den Hoogen, B.G.; Herfst,S.; Sprong,L.; Cane,P.A.; Forleo-Neto,E.; deSwart,R.L.; Osterhaus,A.D.; Fouchier,R.A. Antigenic and genetic variability of human metapneumoviruses. Emerg. Infect. Dis.2004,10,658-666.

7. Xiao, Ni-guang. Virus profile of hospitalized children with acute lower respiratory tract infection and status analysis of human metapneumovirus in Changsha area[D].University of south China, 2011.

8. Aberle J H, Aberle S W, Redlberger-Fritz M, Sandhofer M J, Popow T. Human metapneumovirus subgroup changes and seasonality during epidemics $\llbracket J$ J. Pediatr Infect Dis J, 2010,29(11):1016-1018.

9. Zhu R N, Qian Y, Zhao L Q, Deng J, Sun Y, Wang F, Liao B, Li Y, Huang R Y. Characterization of human metapneumovirus from pediatric patients with acute respiratory infections in a 4-year period in Beijing,

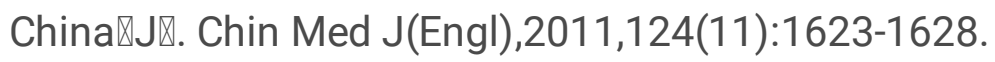

10. Kong W, Wang Y, Zhu H, Lin X, Yu B, Hu Q, Yang X, G uo D, Peng J, Zhou D. Circulation of human metapneumovirus among children with influenza-like illness in Wuhan, China囚J囚. Journal of Medical Virology, 2016,88(5):774-781.

11. Zeng S, Xiao N, Zhong L, Yu T, Zhang B, Duan Z J. Clinical features of human metapneumovirus genotypes in children with acute lower respiratory tract infection in Changsha, China $\mathbb{J} \mathrm{J}$. Journal of Medical Virology,2015,87(11):1839-1845.

12.Zhang, L. et al. Epidemiological and clinical features of human metapneumovirus in hospitalised paediatric patients with acute respiratory illness: a cross-sectional study in Southern China, from 2013 to 2016. BMJ Open8, e019308 (2018).

15.Li, X. et al. Viral etiologies and epidemiology of patients with acute respiratory infections based on sentinel hospitals in Gansu Province, Northwest China, 2011-2015. J. Med. Virol.90, 828-835 (2018). 
16.Peiris, J. S. M. et al. Children with Respiratory Disease Associated with Metapneumovirus in Hong Kong. Emerging Infectious Diseases9, 628-633 (2003).

17.Zhang, C. et al. Detection and Genetic Diversity of Human Metapneumovirus in Hospitalized Children with Acute Respiratory Infections in Southwest China. Journal of Clinical Microbiology50, 2714-2719 (2012).

18.Saikusa, M. et al. 180-Nucleotide Duplication in the G Gene of Human metapneumovirus A2b Subgroup Strains Circulating in Yokohama City, Japan, since 2014. Frontiers in Microbiology8 (2017).

19.Saikusa, $\mathrm{M}$. et al. A novel 111-nucleotide duplication in the $\mathrm{G}$ gene of human metapneumovirus: HMPV evolution. Microbiology and Immunology61, 507-512 (2017).

20. Ren J, Phan T, Bao X, Recent vaccine development for human metapneumovirus. J Gen Virol, 2015,96(Pt 7):1515-1520.

21. Ministry of Health of the People ${ }^{\square}$ Republic of China.Circular of the General Office of the Ministry of Health on the issuance of the sentinel surveillance programme (2011 edition) for hospitalized cases of severe acute respiratory infections(EB/OL). (2011-02-11) 『2014-11-17区, Http//www.gov.cn/zwgk/201102/11/content_1801649.htm.

22.Panda, S., Mohakud, N. K., Pena, L. \& Kumar, S. Human metapneumovirus: review of an important respiratory pathogen. International Journal of Infectious Diseases25, 45-52 (2014).

23.Naz, R. et al. Etiology of acute viral respiratory infections common in Pakistan: A review. Rev Med Virol e2024, https://doi.org/10.1002/rmv.2024 (2018)

24. .Williams JV, Edwards KM, Weinberg GA, Griffin MR, Hall CB, Zhu Y, Szilagyi PG, Wang CK, Yang C-F, Silva $\mathrm{D}$, et al. Population-based incidence of human metapneumovirus infection among hospitalized children. J Infect Dis. 2010;201:1890-8.

25..Bastien N, Ward D, Van Caeseele P, Brandt K, Lee SHS, McNabb G, Klisko B, Chan E, Li Y. Human metapneumovirus infection in the Canadian population. J Clin Microbiol. 2003;41(10):4642-6.

26. Mullins JA, Erdman DD, Weinberg GA, Edwards K, Hall CB, Walker FJ,I wane M, Anderson LJ. Human metapneumovirus infection among children hospitalized with acute respiratory illness. Emerg Infect Dis. 2004;10(4).

27.Mackay IM, Bialasiewicz S, Jacob KC, McQueen E, Arden KE, Nissen MD, Sloots TP. "Genetic diversity of human metapneumovirus over 4 consecutive years in Australia”. J Infect Dis. 2006;193(12):1630-3.

28. Banerjee S, Sullender WM, Choudekar A, John C, Tyagi V, Fowler K, Lefkowitz EJ. Detection and genetic diversity of human metapneumovirus in hospitalized children with acute respiratory infections in India. J Clin Virol.2011;83:1799-810. 
29. Williams JV, Edwards KM, Weinberg GA, Griffin MR, Hall CB, Zhu Y, Szilagyi PG, Wang CK, Yang C-F, Silva $D$, et al. Population-based incidence of human metapneumovirus infection among hospitalized children. J Infect Dis.2010;201:1890-8.

30. Naganori Nao, Miwako Saikusa, KoSato, Tsuyoshi Sekizuka, Shuzo Usuku, Nobuko Tanaka, Hidekazu Nishimura and Makoto Takeda. Recent Molecular Evolution of Human Metapneumovirus (HMPV): Subdivision of HMPVA 2b Strains. Microorganisms 2020, 1280(8);

doi:10.3390/microorganisms8091280.

31. LIUShiwen『LIJianxiong $₫ G O N G T i a n$.et al. Analysis of human metapneumovirus infection in 2438 children with acute respiratory tract infection in Jiangxi province. Experimental and Laboratory Medicine, 2019,37(4):562-564,589.

\section{Tables}

Table 1. hMPV-positive in Pediatric Patients of different ages and gender with SARI

\begin{tabular}{|c|c|c|c|c|c|c|}
\hline Variable & $\begin{array}{l}\text { Tested SARI } \\
\text { cases } \\
\mathrm{N} \text { (percentage) }\end{array}$ & $\begin{array}{l}\text { hMPV-positive } \\
\text { cases } \\
\text { N(percentage) }\end{array}$ & $\begin{array}{l}\text { hMPV-negtive } \\
\text { cases } \\
\mathrm{N} \text { (percentage) }\end{array}$ & $\begin{array}{l}\text { Positive } \\
\text { rate }\end{array}$ & $\chi^{2}$ & $P$ \\
\hline Gender & & & & & 0.724 & 0.395 \\
\hline Male & $605 \rrbracket 53.40 \rrbracket$ & $33 \llbracket 58.93 \rrbracket$ & $572(53.11)$ & $5.45 \%$ & & \\
\hline Female & $528 \rrbracket 46.60 \rrbracket$ & $23 \rrbracket 41.07 \rrbracket$ & $505(46.29)$ & $4.36 \%$ & & \\
\hline $\begin{array}{l}\text { Age } \\
\text { (years) }\end{array}$ & & & & & 1.126 & 0.890 \\
\hline OQ & $315 \rrbracket 27.80 \rrbracket$ & 19ه33.93》 & $296 \rrbracket 27.48 \rrbracket$ & $6.03 \%$ & & \\
\hline 1区 & $361 \rrbracket 31.86 \rrbracket$ & $16 \rrbracket 28.57 \rrbracket$ & $345 \rrbracket 32.03 \rrbracket$ & $4.43 \%$ & & \\
\hline 38 & $282 \varangle 24.89 \rrbracket$ & $13 \llbracket 23.21 \rrbracket$ & $269 ه 24.98 \rrbracket$ & $4.61 \%$ & & \\
\hline 5》 & $84 \llbracket 7.41 \rrbracket$ & $4 \rrbracket 7.14 \rrbracket$ & $80 \rrbracket 7.42 \rrbracket$ & $4.76 \%$ & & \\
\hline $7 \bigotimes 14$ & $91 \rrbracket 8.03 \rrbracket$ & $4 \bigotimes 7.14 \rrbracket$ & $87 \rrbracket 8.08 \rrbracket$ & $4.39 \%$ & & \\
\hline Total & 1133 & 56 & 1077 & $4.94 \%$ & & \\
\hline
\end{tabular}

\section{Figures}


sample cases $\longrightarrow$ Hmpv positive rate

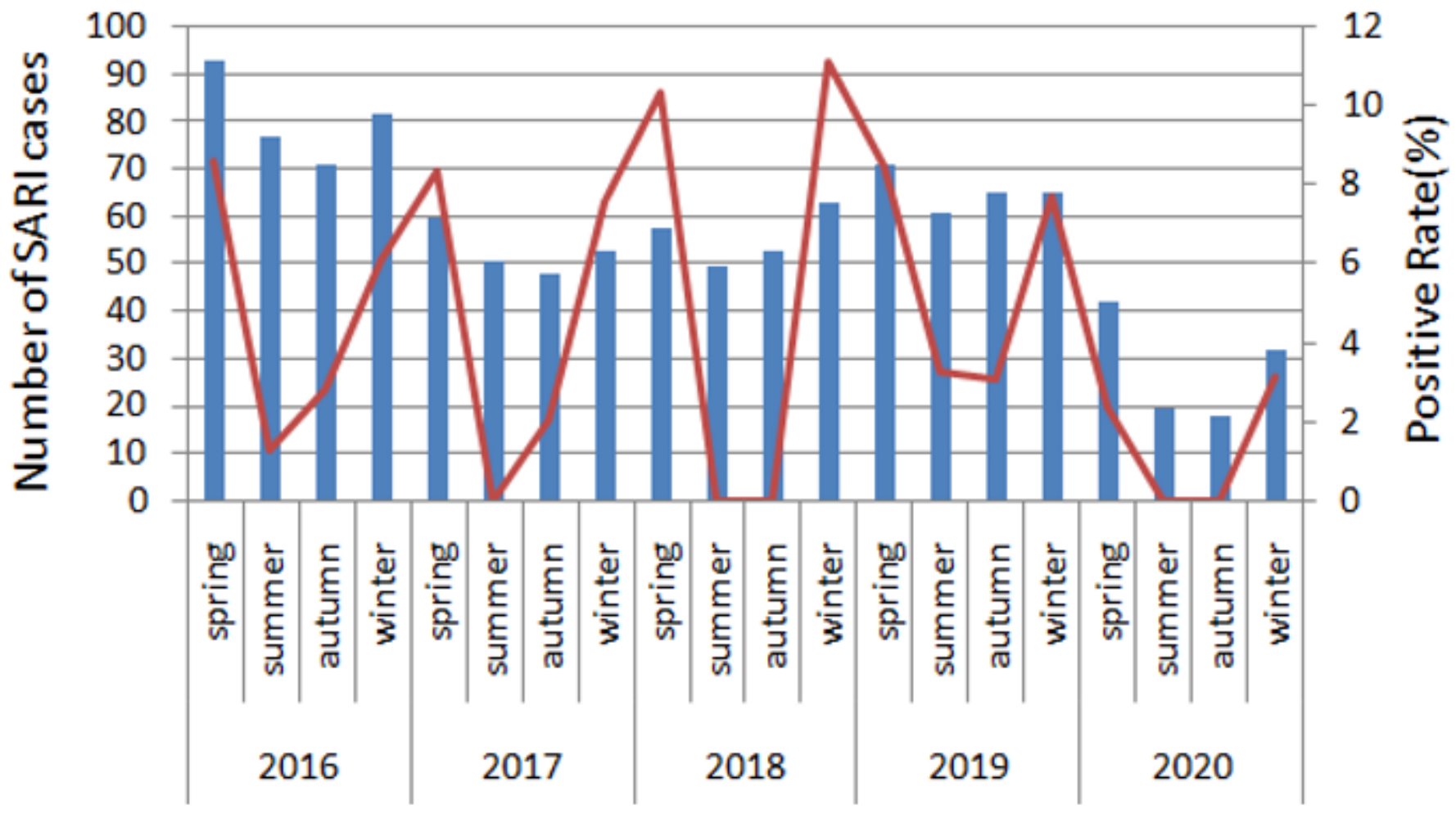

Figure 1

Seasonal distribution of hMPV infections from 2016 to 2020 


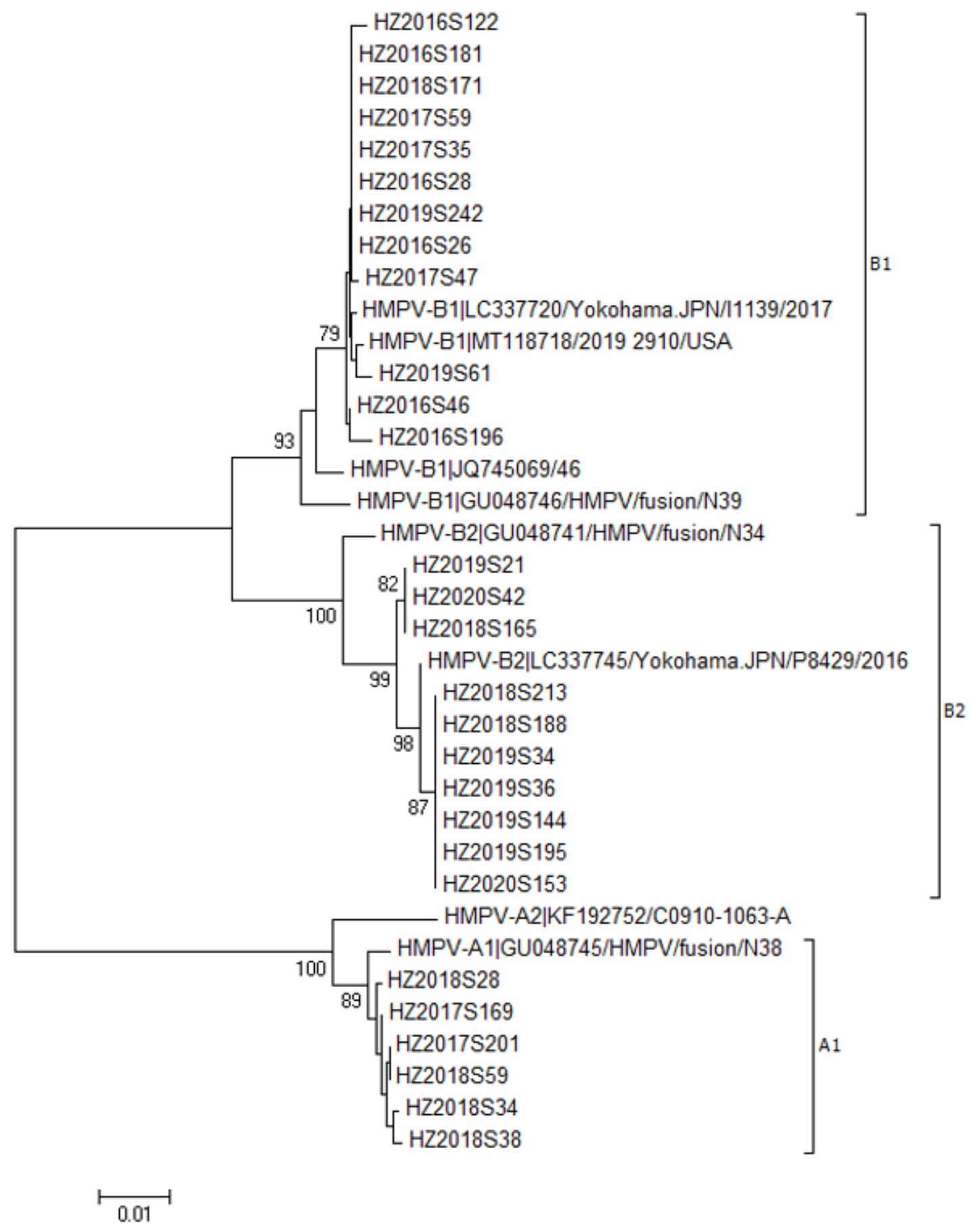

\section{Figure 2}

Genotyping and phylogenetic analysis of hMPV strains. The trees were generated using the neighborjoining method, validated by 1000 bootstrap replicates. Bootstrap values $\geq 70 \%$ are shown on the branch. hMPV sequences identified in this study are indicated by closed circles.

\section{Supplementary Files}


This is a list of supplementary files associated with this preprint. Click to download.

- sequencedata.txt 